\title{
Iron(II) Spin Crossover complexes based on a redox active equatorial Schiff base-like ligand
}

Sophie Schönfeld, ${ }^{a}$ Katja Dankhoff, ${ }^{a}$ Dirk Baabe, ${ }^{b}$ Marc-Kevin Zaretzke, ${ }^{b}$ Martin Bröring, ${ }^{b}$ Konstantin Schötz, ${ }^{c}$ Anna Köhler, ${ }^{c}$ Gerald Hörner, ${ }^{a}$ Birgit Weber ${ }^{a *}$

a Inorganic Chemistry IV, University of Bayreuth, Universitätsstraße 30, 95448 Bayreuth, Germany. * weber@uni-bayreuth.de

${ }^{\mathrm{b}}$ Institute of Inorganic and Analytical Chemistry, Technical University of Braunschweig, Hagenring 30, 38106 Braunschweig, Germany

${ }^{\mathrm{C}}$ Experimental Physics, University of Bayreuth, Universitätsstraße 30, 95448 Bayreuth, Germany

\section{Cartesian coordinates of the DFT optimized structures of ligands}

Table S1: ${ }^{\text {DDLH}}$ (singlet; COSMO(MeCN))

6.177371

6.525292

4.535528

6.568387

2. 075719

5.180060

4.017361

5.745462

3. 806687

2. 735448

4.368818

6.601763

5.738459

5.619480

6.459184

4. 308272

3. 163671

2. 922417

3. 262461

2. 661947

3. 867544

4. 111185

6.977376

3. 025083

7. 197571

6.344950

3. 713286

2. 710190

7. 984128
5.792927

3. 646580

2. 899045

4. 570518

3. 986306

3. 554225

2. 120905

3. 962740

3. 283972

3. 138513

2. 431751

6.055518

3. 906927

3. 232070

5.075436

3. 860802

1.052633

0.814535

4. 275542

0.281359

1. 645154

1.879475

5. 214279

0.576148

7. 417201

4. 391255

5.049774

5. 511243

6.100876
6.204232

9. 443510

7. 965859

1. 659654

11.003482

5.353193

6. 903171

10.369237

9.009332

8.928474

5.579060

5.057059

4.197616

3. 359348

3. 990524

10.169227

7. 168185

8.197396

11.116045

6.128001

4.540444

3. 512524

2. 619418

4. 815593

4. 814197

11.683220

12.120453

13.056707

2. 504944

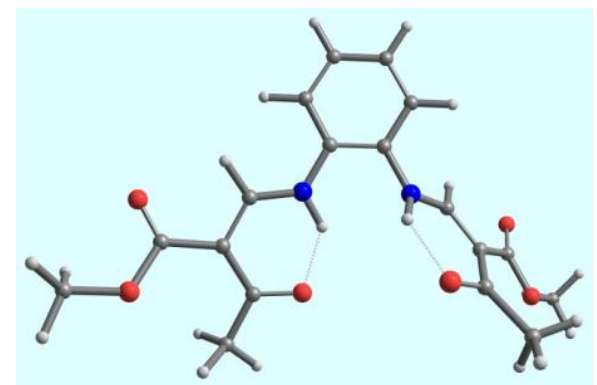




$\begin{array}{rrr}8.488477 & 6.300356 & 1.160083 \\ 7.409984 & 4.161794 & 11.658963 \\ 5.865150 & 3.892808 & 12.524809 \\ 6.202437 & 5.465006 & 11.826098 \\ 8.285929 & 7.342424 & 4.751289 \\ 6.925734 & 8.058754 & 5.652093 \\ 6.850152 & 7.848214 & 3.875682 \\ 5.346196 & 4.236910 & 6.107212 \\ 5.546633 & 3.065571 & 8.083979 \\ 3.260335 & 6.094688 & 13.790221 \\ 2.214915 & 4.662847 & 13.527094 \\ 1.978082 & 6.130609 & 12.538294 \\ 9.254905 & 7.064336 & 1.257870 \\ 7.684128 & 6.637851 & 0.507121 \\ 8.913288 & 5.371902 & 0.779871 \\ 2.006660 & -0.553225 & 6.343699 \\ 2.645065 & -0.022535 & 3.997325\end{array}$

Table S2: ${ }^{\text {TTF }} \mathrm{LH}_{2}$ ( $=3$ ) (singlet; $\mathrm{COSMO}(\mathrm{MeCN})$ )

\begin{tabular}{|c|c|c|c|}
\hline 0 & 6.860711 & 5.769279 & 6.645503 \\
\hline 0 & 6.456340 & 3.268162 & 9.232484 \\
\hline $\mathrm{N}$ & 4.243042 & 3.368132 & 7.940008 \\
\hline 0 & 9.366140 & 5.169747 & 3.312735 \\
\hline 0 & 2.342654 & 3.901508 & 11.476740 \\
\hline $\mathrm{N}$ & 5.250324 & 4.134564 & 5.494186 \\
\hline $\mathrm{C}$ & 3.519307 & 3.054547 & 6.777762 \\
\hline C & 5.859761 & 3.413308 & 10.323110 \\
\hline C & 3.700857 & 3.545378 & 9.142583 \\
\hline $\mathrm{H}$ & 2.621781 & 3.639036 & 9.186875 \\
\hline $\mathrm{C}$ & 4.050105 & 3.409305 & 5.524673 \\
\hline $\mathrm{C}$ & 7.641389 & 5.713165 & 5.669983 \\
\hline C & 6.132862 & 4.148555 & 4.497080 \\
\hline $\mathrm{H}$ & 5.917400 & 3.519814 & 3.643487 \\
\hline C & 7.309736 & 4.889075 & 4.515932 \\
\hline C & 4.416705 & 3.621025 & 10.330237 \\
\hline $\mathrm{C}$ & 2.323149 & 2.344884 & 6.856830 \\
\hline $\mathrm{H}$ & 1.943884 & 2.029043 & 7.819574 \\
\hline $\mathrm{C}$ & 3.568095 & 3.852203 & 11.509809 \\
\hline $\mathrm{C}$ & 1.666061 & 1.984470 & 5.692674 \\
\hline S & 0.129452 & 1.103702 & 5.683398 \\
\hline C & 3.386421 & 3.034429 & 4.356335 \\
\hline $\mathrm{H}$ & 3.780799 & 3.321127 & 3.390558 \\
\hline C & 8.212461 & 4.770053 & 3.365620 \\
\hline $\mathrm{C}$ & 2.207047 & 2.309955 & 4.443747 \\
\hline S & 1.274074 & 1.782366 & 3.028481 \\
\hline C & 8.914091 & 6.512338 & 5.726981 \\
\hline $\mathrm{H}$ & 9.775165 & 5.841473 & 5.756635 \\
\hline $\mathrm{H}$ & 9.032543 & 7.133643 & 4.838144 \\
\hline $\mathrm{H}$ & 8.889656 & 7.129901 & 6.624280 \\
\hline C & 6.674971 & 3.334180 & 11.585854 \\
\hline $\mathrm{H}$ & 6.243489 & 2.624348 & 12.292269 \\
\hline $\mathrm{H}$ & 7.687270 & 3.036483 & 11.315757 \\
\hline $\mathrm{H}$ & 6.693820 & 4.305138 & 12.085247 \\
\hline 0 & 4.254182 & 4.017212 & 12.654790 \\
\hline C & 3.452768 & 4.188128 & 13.848358 \\
\hline 0 & 7.628377 & 4.143295 & 2. 311328 \\
\hline C & 8.487465 & 3.919127 & 1.168285 \\
\hline C & 0.263078 & 0.678307 & 3.971483 \\
\hline S & -0.279342 & -0.867649 & 1.748923 \\
\hline $\mathrm{C}$ & -0.367510 & -0.383758 & 3.445799 \\
\hline S & -1.387297 & -1.449740 & 4.422746 \\
\hline C & -0.746228 & -2.553888 & 2.077312 \\
\hline C & -1.238397 & -2.821531 & 3.303159 \\
\hline S & -0.589579 & -3.689484 & 0.742543 \\
\hline $\mathrm{C}$ & -2.352966 & -3.977336 & 0.309467 \\
\hline S & -1.907957 & -4.368759 & 3.782399 \\
\hline C & -1.290357 & -4.513832 & 5.504813 \\
\hline $\mathrm{H}$ & -2.337047 & -4.601247 & -0.583634 \\
\hline $\mathrm{H}$ & -2.835280 & -3.026839 & 0.093502 \\
\hline $\mathrm{H}$ & -2.860165 & -4.501285 & 1.116996 \\
\hline $\mathrm{H}$ & -1.616441 & -5.497758 & 5.838783 \\
\hline $\mathrm{H}$ & -1.733971 & -3.747784 & 6.136321 \\
\hline $\mathrm{H}$ & -0.204372 & -4.452525 & 5.513833 \\
\hline $\mathrm{H}$ & 2.848147 & 5.090757 & 13.767097 \\
\hline $\mathrm{H}$ & 4.173574 & 4.275905 & 14.656536 \\
\hline
\end{tabular}

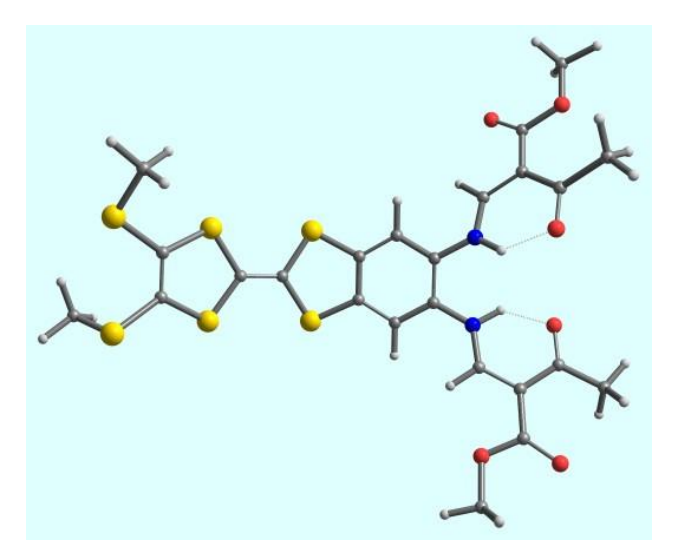




$\begin{array}{llll}\mathrm{H} & 2.809321 & 3.320671 & 13.993077 \\ \mathrm{H} & 7.853554 & 3.430745 & 0.432844 \\ \mathrm{H} & 9.321259 & 3.275769 & 1.448523 \\ \mathrm{H} & 8.862384 & 4.868610 & 0.787501 \\ \mathrm{H} & 5.528704 & 4.739767 & 6.285194 \\ \mathrm{H} & 5.274765 & 3.300601 & 7.970201\end{array}$

Table S3: ${ }^{\mathrm{ND}} \mathrm{LH}_{2}$ (singlet; COSMO(MeCN))

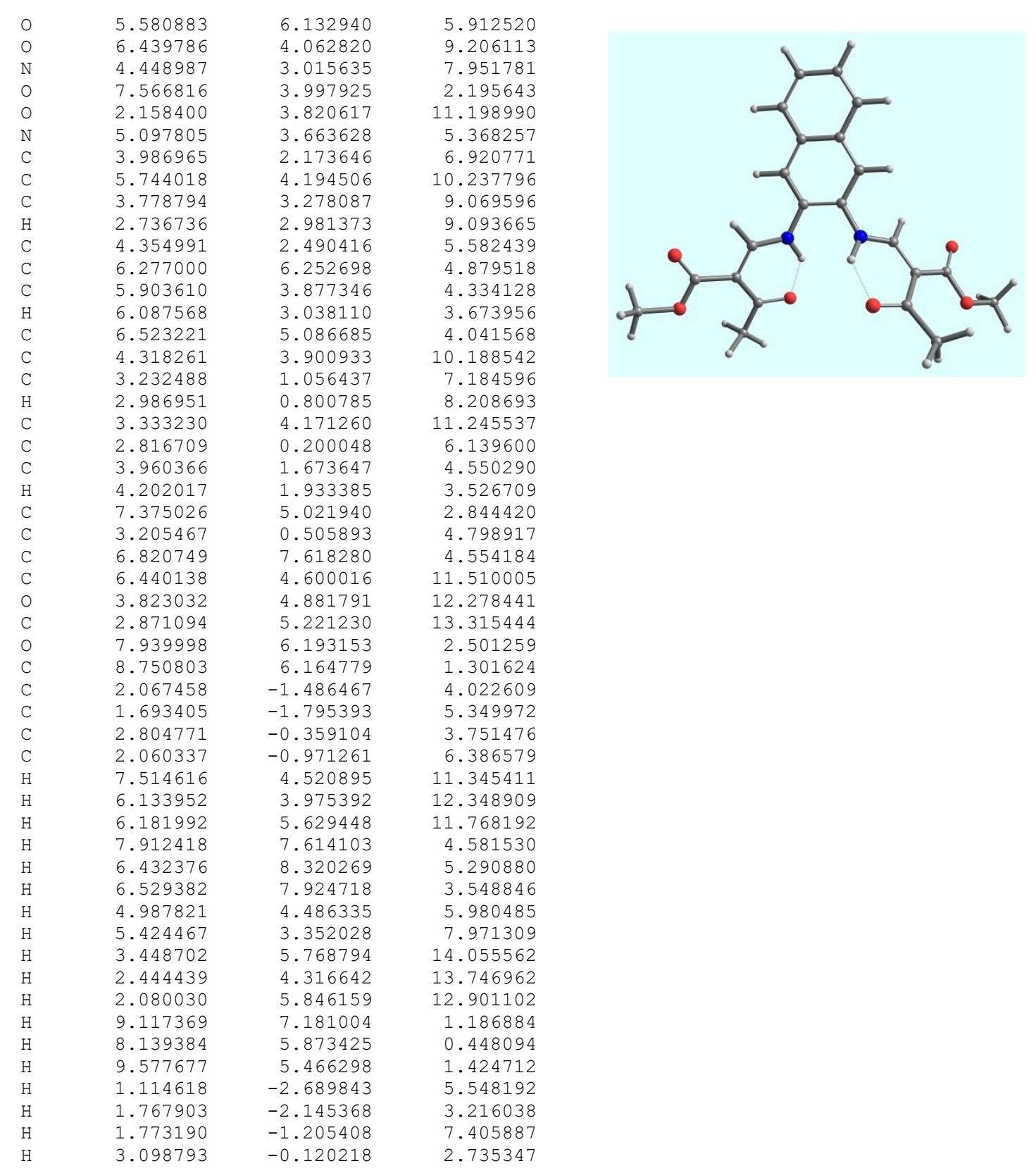


Table S4: ${ }^{\mathrm{PA}} \mathrm{LH}_{2}$ (singlet; COSMO(MeCN))

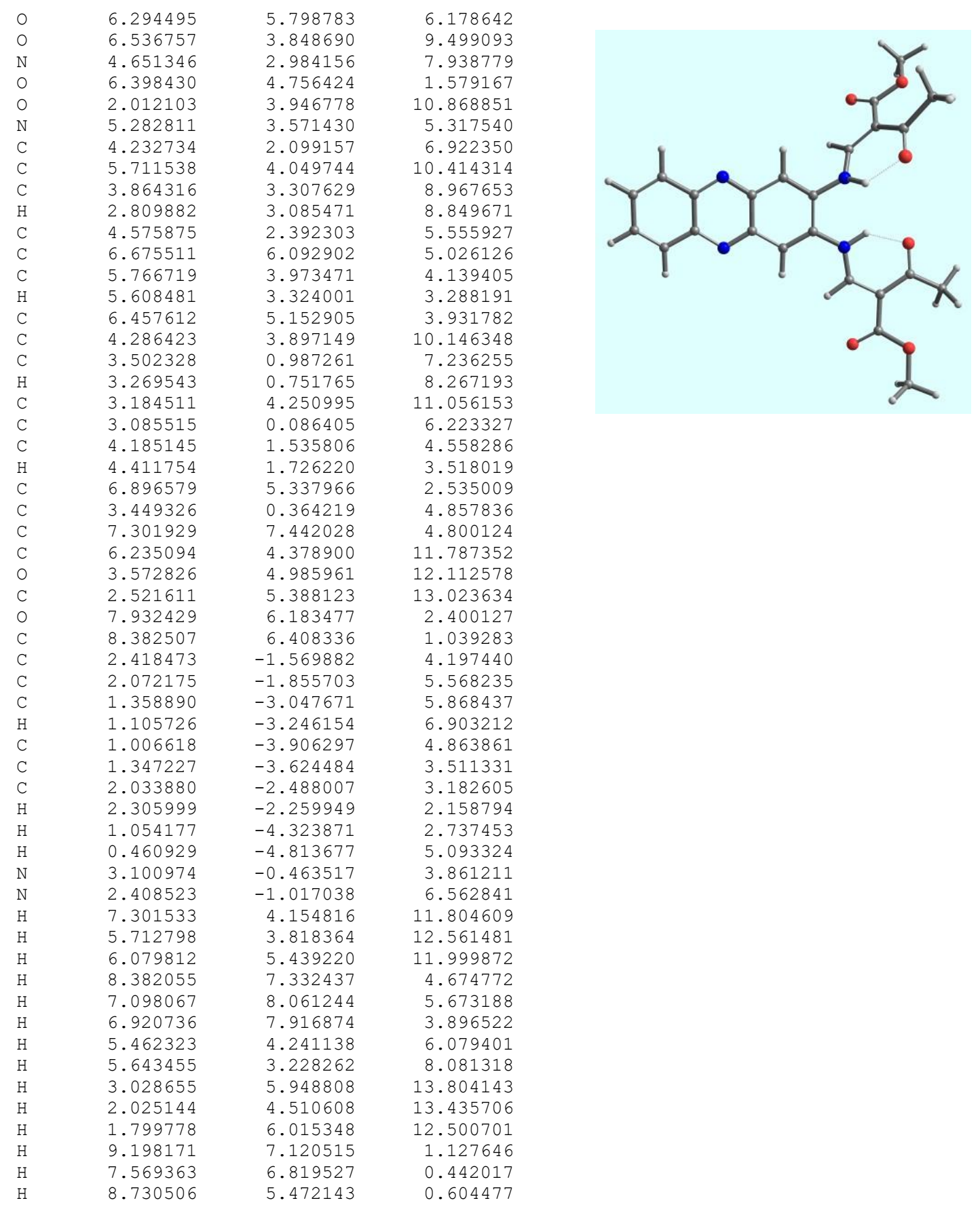

Cartesian coordinates of the DFT optimized structures of iron(II) complexes

Table S5: low spin [Fe $\left.{ }^{\mathrm{PD}} \mathrm{L}(\mathrm{py})_{2}\right]$ (三 low spin REF) (singlet; $\mathrm{COSMO}(\mathrm{MeCN})$ )

$\begin{array}{lllr}\mathrm{Fe} & 6.150939 & 4.012756 & 7.556701 \\ \mathrm{O} & 7.841823 & 4.815390 & 7.033280 \\ \mathrm{O} & 6.631690 & 4.422722 & 9.394702 \\ \mathrm{~N} & 4.497191 & 3.213873 & 8.040168 \\ \mathrm{O} & 8.847406 & 6.048656 & 3.124914 \\ \mathrm{O} & 2.643479 & 3.112831 & 11.677902 \\ \mathrm{~N} & 5.660715 & 3.585263 & 5.772671 \\ \mathrm{~N} & 5.314098 & 5.778573 & 7.416141 \\ \mathrm{C} & 3.868370 & 2.560887 & 6.956101 \\ \mathrm{C} & 5.884262 & 4.358282 & 10.434313 \\ \mathrm{C} & 3.951359 & 3.274735 & 9.238261 \\ \mathrm{H} & 2.954731 & 2.846830 & 9.383256\end{array}$




\begin{tabular}{|c|c|c|}
\hline 4.508163 & 2.768373 & 5.703892 \\
\hline 8.238246 & 5.104553 & 5.849844 \\
\hline 6.288085 & 4.004580 & 4.690989 \\
\hline 5.860287 & 3.757391 & 3.716871 \\
\hline 7.493687 & 4.767244 & 4.667415 \\
\hline 4.538860 & 3.858368 & 10.398588 \\
\hline 2.724665 & 1.748144 & 7.028070 \\
\hline 2.241395 & 1.559016 & 7.986949 \\
\hline 3.639423 & 3.837806 & 11.568997 \\
\hline 2.209917 & 1.160523 & 5.869865 \\
\hline 3.986746 & 2.161764 & 4.548722 \\
\hline 4.479963 & 2.290385 & 3.584567 \\
\hline 7.987720 & 5.193762 & 3.347366 \\
\hline 2.840331 & 1.367044 & 4.631593 \\
\hline 9.580774 & 5.795562 & 5.795018 \\
\hline 10.247209 & 5.324541 & 5.058700 \\
\hline 9.458148 & 6.842057 & 5.477878 \\
\hline 10.028646 & 5.761933 & 6.797516 \\
\hline 6.570065 & 4.790724 & 11.711304 \\
\hline 6.358201 & 4.104781 & 12.542538 \\
\hline 7.651031 & 4.839729 & 11.521576 \\
\hline 6.211313 & 5.784826 & 12.018258 \\
\hline 3.965492 & 4.739179 & 12.537839 \\
\hline 3.132558 & 4.707805 & 13.726124 \\
\hline 7.372582 & 4.531137 & 2.310172 \\
\hline 7.756755 & 4.968254 & 0.982391 \\
\hline 4.169047 & 8.339909 & 7.235994 \\
\hline 5.416964 & 8.151299 & 7.841956 \\
\hline 5.977735 & 8.988908 & 8.258037 \\
\hline 6.920040 & 6.669014 & 8.374713 \\
\hline 5.954343 & 6.868977 & 7.915063 \\
\hline 4.103485 & 5.965597 & 6.829177 \\
\hline 3.506567 & 7.219017 & 6.722769 \\
\hline 2.531937 & 7.305591 & 6.241874 \\
\hline 3.618393 & 5.072383 & 6.440711 \\
\hline 7.046950 & 2.253411 & 7.730158 \\
\hline 7.889272 & 1.800351 & 6.768530 \\
\hline 6.829651 & 1.465557 & 8.812734 \\
\hline 6.142464 & 1.860253 & 9.559421 \\
\hline 7.440273 & 0.223352 & 8.969641 \\
\hline 7.227516 & -0.367994 & 9.860997 \\
\hline 8.032545 & 2.457724 & 5.912013 \\
\hline 8.535504 & 0.569189 & 6.858437 \\
\hline 9.202386 & 0.255968 & 6.054268 \\
\hline 8.310732 & -0.241185 & 7.977117 \\
\hline 8.800815 & -1.211009 & 8.072356 \\
\hline 3.724920 & 9.334011 & 7.165433 \\
\hline 2.094099 & 4.963134 & 13.470625 \\
\hline 3.563247 & 5.460191 & 14.396584 \\
\hline 3.161927 & 3.710468 & 14.188483 \\
\hline 7.161766 & 4.356225 & 0.295733 \\
\hline 8.831274 & 4.800764 & 0.819189 \\
\hline 7.528599 & 6.035765 & 0.848169 \\
\hline 1.323972 & 0.526617 & 5.935940 \\
\hline 2.446939 & 0.895229 & 3.72976 \\
\hline
\end{tabular}

Table S6: high spin [Fe $\left.{ }^{\mathrm{PD}} \mathrm{L}(\mathrm{py})_{2}\right]$ ( (high spin REF) (quintet; $\mathrm{COSMO}(\mathrm{MeCN})$ )

$\begin{array}{lllr}\mathrm{Fe} & 6.447622 & 3.835724 & 7.673739 \\ \mathrm{O} & 8.092701 & 4.562382 & 6.762303 \\ \mathrm{O} & 6.594163 & 4.097293 & 9.668393 \\ \mathrm{~N} & 4.538697 & 3.084778 & 8.073546 \\ \mathrm{O} & 8.288082 & 6.507203 & 3.016037 \\ \mathrm{O} & 2.196160 & 3.553224 & 11.405313 \\ \mathrm{~N} & 5.740846 & 3.459569 & 5.742169 \\ \mathrm{~N} & 5.616938 & 5.900111 & 7.554435 \\ \mathrm{C} & 3.961044 & 2.423864 & 6.974411 \\ \mathrm{C} & 5.703458 & 4.267456 & 10.577074 \\ \mathrm{C} & 3.840490 & 3.335260 & 9.161119 \\ \mathrm{H} & 2.769449 & 3.093009 & 9.167241 \\ \mathrm{C} & 4.605810 & 2.629372 & 5.719669 \\ \mathrm{C} & 8.252430 & 5.097115 & 5.605008 \\ \mathrm{C} & 6.159031 & 4.066122 & 4.650779 \\ \mathrm{H} & 5.550330 & 3.979783 & 3.743241 \\ \mathrm{C} & 7.326541 & 4.885141 & 4.534682 \\ \mathrm{C} & 4.327917 & 3.941892 & 10.359512\end{array}$




$\begin{array}{rrr}2.852799 & 1.562470 & 7.048120 \\ 2.384053 & 1.369021 & 8.014637 \\ 3.268363 & 4.166287 & 11.364837 \\ 2.363910 & 0.933761 & 5.898748 \\ 4.117246 & 1.969989 & 4.577966 \\ 4.632417 & 2.090815 & 3.623533 \\ 7.554796 & 5.541454 & 3.234252 \\ 2.997144 & 1.136960 & 4.663124 \\ 9.501991 & 5.934249 & 5.454270 \\ 10.051179 & 5.688293 & 4.534337 \\ 9.236564 & 7.000411 & 5.377971 \\ 10.136203 & 5.772792 & 6.336930 \\ 6.237459 & 4.775535 & 11.899450 \\ 5.776506 & 4.267689 & 12.756454 \\ 7.326525 & 4.628939 & 11.908543 \\ 6.016916 & 5.849102 & 12.008725 \\ 3.545640 & 5.164956 & 12.248927 \\ 2.557466 & 5.367726 & 13.292551 \\ 6.841598 & 4.957129 & 2.213838 \\ 6.965337 & 5.606145 & 0.922964 \\ 4.583683 & 8.485826 & 7.338633 \\ 5.849621 & 8.270562 & 7.894473 \\ 6.459983 & 9.099901 & 8.253209 \\ 7.308544 & 6.735668 & 8.403620 \\ 6.327079 & 6.961272 & 7.982911 \\ 4.399892 & 6.110754 & 7.019132 \\ 3.843966 & 7.385053 & 6.892716 \\ 2.853716 & 7.507462 & 6.453172 \\ 3.870395 & 5.216462 & 6.685482 \\ 7.294246 & 1.852208 & 7.814170 \\ 8.104424 & 1.368270 & 6.847296 \\ 6.885691 & 1.010285 & 8.789112 \\ 6.223801 & 1.446742 & 9.539344 \\ 7.272082 & -0.328812 & 8.837883 \\ 6.914021 & -0.967322 & 9.646575 \\ 8.400761 & 2.086175 & 6.080386 \\ 8.536910 & 0.042048 & 6.822007 \\ 9.192027 & -0.299571 & 6.019282 \\ 8.114108 & -0.826530 & 7.835591 \\ 8.434144 & -1.869695 & 7.844180 \\ 4.179939 & 9.496457 & 7.253832 \\ 1.595108 & 5.666357 & 12.853677 \\ 2.963466 & 6.170473 & 13.918800 \\ 2.423118 & 4.446196 & 13.877372 \\ 6.321638 & 5.029471 & 0.249175 \\ 1.503711 & 5.579566 & 0.579266 \\ 2.632343 & 6.651007 & 0.983751 \\ 6.265516 & 5.972179 \\ 6.628701 & 3.768696\end{array}$

Table S7: low spin [Fe $\left.{ }^{\mathrm{TTF}} \mathrm{L}(\mathrm{py})_{2}\right]$ (三 low spin 4) (singlet; COSMO(MeCN))

$\begin{array}{lrrr}\mathrm{Fe} & 6.161862 & 3.989451 & 7.557691 \\ \mathrm{O} & 7.843348 & 4.798115 & 7.042080 \\ \mathrm{O} & 6.640253 & 4.402680 & 9.388179 \\ \mathrm{~N} & 4.511260 & 3.196555 & 8.039470 \\ \mathrm{O} & 8.839874 & 6.063935 & 3.142558 \\ \mathrm{O} & 2.585368 & 3.217717 & 11.617460 \\ \mathrm{~N} & 5.674494 & 3.566462 & 5.777696 \\ \mathrm{~N} & 5.328228 & 5.750394 & 7.419635 \\ \mathrm{C} & 3.892491 & 2.534460 & 6.961460 \\ \mathrm{C} & 5.885040 & 4.364443 & 10.423288 \\ \mathrm{C} & 3.954288 & 3.282528 & 9.230906 \\ \mathrm{H} & 2.952734 & 2.868157 & 9.378563 \\ \mathrm{C} & 4.533509 & 2.741292 & 5.709413 \\ \mathrm{C} & 8.230762 & 5.110008 & 5.861648 \\ \mathrm{C} & 6.293688 & 4.001283 & 4.697232 \\ \mathrm{H} & 5.868870 & 3.756920 & 3.721545 \\ \mathrm{C} & 7.486056 & 4.779058 & 4.678833 \\ \mathrm{C} & 4.534923 & 3.880054 & 10.383758 \\ \mathrm{C} & 2.761007 & 1.710132 & 7.047835 \\ \mathrm{H} & 2.287460 & 1.511990 & 8.008613 \\ \mathrm{C} & 3.618950 & 3.891301 & 11.538214 \\ \mathrm{C} & 2.259256 & 1.122762 & 5.888818 \\ \mathrm{~S} & 0.796603 & 0.102577 & 5.873893 \\ \mathrm{C} & 4.029860 & 2.128985 & 4.551970\end{array}$




\begin{tabular}{|c|c|c|c|}
\hline $\mathrm{H}$ & 4.529265 & 2.250930 & 3.591358 \\
\hline C & 7.969283 & 5.220512 & 3.362290 \\
\hline C & 2.890303 & 1.332396 & 4.645549 \\
\hline S & 2.131763 & 0.555169 & 3.230301 \\
\hline C & 9.559096 & 5.822653 & 5.815914 \\
\hline $\mathrm{H}$ & 10.245511 & 5.363635 & 5.092627 \\
\hline $\mathrm{H}$ & 9.427422 & 6.867266 & 5.501414 \\
\hline $\mathrm{H}$ & 9.994508 & 5.795494 & 6.822193 \\
\hline C & 6.568902 & 4.815371 & 11.691437 \\
\hline $\mathrm{H}$ & 6.371856 & 4.139698 & 12.532565 \\
\hline $\mathrm{H}$ & 7.646385 & 4.869283 & 11.493844 \\
\hline $\mathrm{H}$ & 6.209557 & 5.810240 & 11.989709 \\
\hline 0 & 3.976226 & 4.757455 & 12.526110 \\
\hline C & 3.117583 & 4.767022 & 13.693910 \\
\hline 0 & 7.326162 & 4.586315 & 2.325338 \\
\hline C & 7.698456 & 5.028815 & 0.998217 \\
\hline $\mathrm{C}$ & 4.191277 & 8.313136 & 7.253354 \\
\hline C & 5.438078 & 8.117863 & 7.855780 \\
\hline $\mathrm{H}$ & 6.001397 & 8.951554 & 8.274940 \\
\hline $\mathrm{H}$ & 6.938086 & 6.636263 & 8.380368 \\
\hline C & 5.972178 & 6.835572 & 7.922383 \\
\hline C & 4.118620 & 5.943243 & 6.835297 \\
\hline $\mathrm{C}$ & 3.526324 & 7.197586 & 6.736001 \\
\hline $\mathrm{H}$ & 2.551825 & 7.289075 & 6.256932 \\
\hline $\mathrm{H}$ & 3.625188 & 5.057614 & 6.441281 \\
\hline $\mathrm{N}$ & 7.051852 & 2.228438 & 7.727500 \\
\hline C & 7.889749 & 1.771773 & 6.764882 \\
\hline C & 6.822765 & 1.432710 & 8.800793 \\
\hline $\mathrm{H}$ & 6.142090 & 1.821323 & 9.555187 \\
\hline $\mathrm{C}$ & 7.413039 & 0.180916 & 8.944949 \\
\hline $\mathrm{H}$ & 7.188997 & -0.415344 & 9.829614 \\
\hline $\mathrm{H}$ & 8.051157 & 2.428234 & 5.912000 \\
\hline C & 8.515465 & 0.531016 & 6.843165 \\
\hline $\mathrm{H}$ & 9.178496 & 0.216956 & 6.036853 \\
\hline $\mathrm{C}$ & 8.276167 & -0.287781 & 7.950427 \\
\hline $\mathrm{H}$ & 8.749730 & -1.266042 & 8.035715 \\
\hline $\mathrm{H}$ & 3.749821 & 9.308187 & 7.188540 \\
\hline C & 1.055705 & -0.474128 & 4.207910 \\
\hline S & 0.661049 & -2.163665 & 2.043060 \\
\hline C & 0.444984 & -1.574603 & 3.711326 \\
\hline S & -0.663787 & -2.592531 & 4.664997 \\
\hline C & 0.206769 & -3.847895 & 2.448649 \\
\hline $\mathrm{C}$ & -0.387542 & -4.049517 & 3.654152 \\
\hline S & 0.531280 & -5.058058 & 1.199346 \\
\hline $\mathrm{C}$ & -1.174146 & -5.483510 & 0.638938 \\
\hline S & -1.070189 & -5.583182 & 4.188791 \\
\hline C & -0.310191 & -5.763457 & 5.858702 \\
\hline $\mathrm{H}$ & -1.043140 & -6.117069 & -0.247302 \\
\hline $\mathrm{H}$ & -1.713884 & -4.570280 & 0.366623 \\
\hline $\mathrm{H}$ & -1.708951 & -6.040199 & 1.416433 \\
\hline $\mathrm{H}$ & -0.652039 & -6.737958 & 6.227558 \\
\hline $\mathrm{H}$ & -0.658149 & -4.973301 & 6.532609 \\
\hline $\mathrm{H}$ & 0.781916 & -5.756369 & 5.774135 \\
\hline $\mathrm{H}$ & 2.105659 & 5.096105 & 13.424262 \\
\hline $\mathrm{H}$ & 3.581189 & 5.480280 & 14.382445 \\
\hline $\mathrm{H}$ & 3.070876 & 3.768464 & 14.147567 \\
\hline $\mathrm{H}$ & 7.079951 & 4.438550 & 0.315773 \\
\hline $\mathrm{H}$ & 8.763386 & 4.837918 & 0.810980 \\
\hline & 7. & 6.0 & 0 . \\
\hline
\end{tabular}

Table S8: high spin $\left[\mathrm{Fe}^{\mathrm{TTF}} \mathrm{L}(\mathrm{py})_{2}\right]$ (三 high spin 4) (quintet; $\operatorname{COSMO}(\mathrm{MeCN})$ )

$\begin{array}{lllr}\mathrm{Fe} & 6.472874 & 3.814957 & 7.677102 \\ \mathrm{O} & 8.100487 & 4.551294 & 6.759128 \\ \mathrm{O} & 6.603526 & 4.079211 & 9.669711 \\ \mathrm{~N} & 4.576352 & 3.055582 & 8.074559 \\ \mathrm{O} & 8.329236 & 6.444574 & 2.995545 \\ \mathrm{O} & 2.141657 & 3.729440 & 11.287053 \\ \mathrm{~N} & 5.777822 & 3.422274 & 5.754918 \\ \mathrm{~N} & 5.641074 & 5.903851 & 7.592143 \\ \mathrm{C} & 4.046075 & 2.335549 & 6.997945 \\ \mathrm{C} & 5.697278 & 4.306399 & 10.550870 \\ \mathrm{C} & 3.857287 & 3.338925 & 9.139615 \\ \mathrm{H} & 2.787996 & 3.091771 & 9.140668 \\ \mathrm{C} & 4.695189 & 2.534493 & 5.743764 \\ \mathrm{C} & 8.244318 & 5.106140 & 5.609951\end{array}$




\begin{tabular}{|c|c|c|}
\hline 6.183028 & 4.025615 & 4.657075 \\
\hline 5.587174 & 3.903841 & 3.746444 \\
\hline 7.320991 & 4.880004 & 4.539997 \\
\hline 4.322623 & 3.996369 & 10.315862 \\
\hline 2.992572 & 1.415076 & 7.103105 \\
\hline 2.531166 & 1.219223 & 8.071204 \\
\hline 3.239731 & 4.294546 & 11.272289 \\
\hline 2.565626 & 0.726020 & 5.968228 \\
\hline 1.197636 & -0.420105 & 5.983818 \\
\hline 4.274191 & 1.810619 & 4.617488 \\
\hline 4.799014 & 1.917635 & 3.668338 \\
\hline 7.525214 & 5.545198 & 3.243472 \\
\hline 3.203026 & 0.924780 & 4.730028 \\
\hline 2.552656 & 0.006671 & 3.343942 \\
\hline 9.463513 & 5.984066 & 5.477918 \\
\hline 10.060316 & 5.730855 & 4.592129 \\
\hline 9.170547 & 7.037480 & 5.360048 \\
\hline 10.065266 & 5.870507 & 6.388138 \\
\hline 6.216858 & 4.864606 & 11.854560 \\
\hline 5.715374 & 4.439271 & 12.730547 \\
\hline 7.295157 & 4.667098 & 11.900769 \\
\hline 6.056782 & 5.953124 & 11.889018 \\
\hline 3.529973 & 5.305045 & 12.136464 \\
\hline 2.512855 & 5.594559 & 13.128283 \\
\hline 6.694508 & 5.056612 & 2.262412 \\
\hline 6.817209 & 5.695562 & 0.969506 \\
\hline 4.600723 & 8.494745 & 7.549144 \\
\hline 5.850609 & 8.240399 & 8.120686 \\
\hline 6.446081 & 9.040334 & 8.560451 \\
\hline 7.300917 & 6.681810 & 8.555630 \\
\hline 6.331965 & 6.931674 & 8.121242 \\
\hline 4.439048 & 6.153082 & 7.041204 \\
\hline 3.881904 & 7.430630 & 6.997985 \\
\hline 2.903577 & 7.582932 & 6.542673 \\
\hline 3.915498 & 5.291496 & 6.625065 \\
\hline 7.317504 & 1.810940 & 7.795311 \\
\hline 8.061280 & 1.321696 & 6.779509 \\
\hline 6.900674 & 0.952561 & 8.751335 \\
\hline 6.298135 & 1.386034 & 9.550434 \\
\hline 7.205749 & -0.406346 & 8.727756 \\
\hline 6.840890 & -1.056555 & 9.523147 \\
\hline 8.373979 & 2.046987 & 6.027413 \\
\hline 8.410109 & -0.023663 & 6.681978 \\
\hline 9.011420 & -0.367339 & 5.839809 \\
\hline 7.972480 & -0.909223 & 7.671660 \\
\hline 8.225358 & -1.969100 & 7.621773 \\
\hline 4.193527 & 9.506836 & 7.533172 \\
\hline 1.532179 & -1.050215 & 4.350935 \\
\hline 1.307993 & -2.856914 & 2.258682 \\
\hline 1.012457 & -2.213580 & 3.894773 \\
\hline-0.042560 & -3.265769 & 4.870721 \\
\hline 0.966266 & -4.550277 & 2.730779 \\
\hline 0.360352 & -4.742641 & 3.931795 \\
\hline 1.411169 & -5.786646 & 1.545390 \\
\hline-0.241909 & -6.356485 & 0.956789 \\
\hline-0.224484 & -6.296120 & 4.521134 \\
\hline 0.507120 & -6.350494 & 6.212317 \\
\hline-0.039133 & -7.015153 & 0.102969 \\
\hline-0.835841 & -5.496201 & 0.630142 \\
\hline-0.760197 & -6.917128 & 1.742590 \\
\hline 0.222229 & -7.328738 & 6.618285 \\
\hline 0.092286 & -5.556673 & 6.842438 \\
\hline 1.598169 & -6.274015 & 6.149240 \\
\hline 1.587825 & 5.931474 & 12.643239 \\
\hline 2.934862 & 6.394765 & 13.744369 \\
\hline 2.305017 & 4.706606 & 13.739534 \\
\hline 6.083184 & 5.193532 & 0.331672 \\
\hline 7.829804 & 5.561663 & 0.565459 \\
\hline 6.591651 & 6.767688 & 1.04323 \\
\hline
\end{tabular}

Table S9: low spin [ $\mathrm{Fe}^{\mathrm{ND}} \mathrm{L}($ py) 2$]$ (singlet; $\mathrm{COSMO}(\mathrm{MeCN})$ )

$\begin{array}{llll}\mathrm{Fe} & 6.049240 & 4.023685 & 7.310195 \\ \mathrm{O} & 7.577428 & 5.011499 & 6.644626 \\ \mathrm{O} & 6.428102 & 4.798281 & 9.044803 \\ \mathrm{~N} & 4.554482 & 3.045605 & 7.936386\end{array}$




\begin{tabular}{|c|c|c|}
\hline 7.164469 & 4.175835 & 1.961647 \\
\hline 2.602579 & 3.322904 & 11.491307 \\
\hline 5.656579 & 3.253649 & 5.625763 \\
\hline 4.080491 & 2.103344 & 6.997466 \\
\hline 5.671506 & 4.804842 & 10.079564 \\
\hline 3.971033 & 3.231574 & 9.102143 \\
\hline 3.051640 & 2.682147 & 9.326064 \\
\hline 4.694500 & 2.222561 & 5.702831 \\
\hline 7.900332 & 5.218396 & 5.421374 \\
\hline 6.177587 & 3.645965 & 4.481794 \\
\hline 5.809005 & 3.200493 & 3.553033 \\
\hline 7.202077 & 4.621060 & 4.320166 \\
\hline 4.422395 & 4.101472 & 10.134331 \\
\hline 3.140907 & 1.113243 & 7.231492 \\
\hline 2.695795 & 0.990400 & 8.219595 \\
\hline 3.500479 & 4.146398 & 11.282937 \\
\hline 2.753859 & 0.222376 & 6.197863 \\
\hline 4.340075 & 1.352988 & 4.684473 \\
\hline 4.818539 & 1.414407 & 3.706479 \\
\hline 7.505090 & 4.897743 & 2.905600 \\
\hline 3.363080 & 0.347316 & 4.897288 \\
\hline 9.116893 & 6.096931 & 5.254369 \\
\hline 6.247001 & 5.566923 & 11.249178 \\
\hline 3.681143 & 5.219434 & 12.102838 \\
\hline 2.819722 & 5.273012 & 13.266729 \\
\hline 8.171549 & 6.067390 & 2.696992 \\
\hline 8.534905 & 6.346878 & 1.322660 \\
\hline 2.027990 & -1.534418 & 4.103087 \\
\hline 1.431086 & -1.659586 & 5.384481 \\
\hline 2.975349 & -0.555520 & 3.867992 \\
\hline 1.788402 & -0.801323 & 6.407915 \\
\hline 7.305345 & 5.762126 & 11.038281 \\
\hline 6.144994 & 5.019294 & 12.194077 \\
\hline 5.725824 & 6.526028 & 11.376767 \\
\hline 9.814074 & 5.706047 & 4.503457 \\
\hline 9.614444 & 6.174308 & 6.228485 \\
\hline 8.821666 & 7.102676 & 4.923795 \\
\hline 7.225446 & 2.486461 & 7.736052 \\
\hline 7.132780 & 1.850197 & 8.929438 \\
\hline 6.399674 & 2.242832 & 9.631185 \\
\hline 8.123443 & 2.020601 & 6.834016 \\
\hline 8.171755 & 2.547882 & 5.883126 \\
\hline 7.919226 & 0.749204 & 9.253835 \\
\hline 8.941448 & 0.924121 & 7.090057 \\
\hline 8.841511 & 0.268203 & 8.320287 \\
\hline 7.798638 & 0.276310 & 10.228381 \\
\hline 9.642332 & 0.591438 & 6.324233 \\
\hline 9.466261 & -0.596879 & 8.545169 \\
\hline 4.926676 & 5.570260 & 6.911701 \\
\hline 3.709718 & 5.457609 & 6.320647 \\
\hline 3.380783 & 4.449407 & 6.077341 \\
\hline 5.367125 & 6.815709 & 7.228870 \\
\hline 6.345679 & 6.859727 & 7.700895 \\
\hline 2.912263 & 6.559488 & 6.032172 \\
\hline 4.620787 & 7.959795 & 6.968341 \\
\hline 3.367788 & 7.840456 & 6.358536 \\
\hline 1.944051 & 6.405130 & 5.556100 \\
\hline 5.025342 & 8.933523 & 7.245071 \\
\hline 2.763392 & 8.721991 & 6.143077 \\
\hline 3.128130 & 6.173108 & 13.808233 \\
\hline 2.958344 & 4.381192 & 13.892189 \\
\hline 1.767279 & 5.349601 & 12.963558 \\
\hline 9.078590 & 7.295905 & 1.359216 \\
\hline 7.637232 & 6.442617 & 0.698410 \\
\hline 9.176543 & 5.550477 & 0.92338 \\
\hline 0.685323 & -2.437246 & 5.559713 \\
\hline 1.736265 & -2.215925 & 3.302020 \\
\hline 1.330032 & -0.895268 & 7.39489 \\
\hline 3.438249 & -0.458243 & 2.88333 \\
\hline
\end{tabular}

Table S10: high spin [ $\left.\mathrm{Fe}^{\mathrm{ND}} \mathrm{L}(\mathrm{py})_{2}\right]$ (quintet; $\mathrm{COSMO}(\mathrm{MeCN})$ )

$\begin{array}{llll}\mathrm{Fe} & 6.458711 & 3.886838 & 7.479947 \\ \mathrm{O} & 7.959460 & 4.716549 & 6.428475 \\ \mathrm{O} & 6.540753 & 4.456001 & 9.406029 \\ \mathrm{~N} & 4.685213 & 2.940473 & 8.010600\end{array}$




\begin{tabular}{|c|c|c|}
\hline 6.463697 & 4.648478 & 1.909364 \\
\hline 2.137208 & 3.777360 & 11.082576 \\
\hline 5.811057 & 3.152682 & 5.644603 \\
\hline 4.271320 & 1.988000 & 7.069212 \\
\hline 5.601035 & 4.701697 & 10.248482 \\
\hline 3.924247 & 3.284633 & 9.026160 \\
\hline 2.899010 & 2.895951 & 9.075789 \\
\hline 4.886737 & 2.106498 & 5.770028 \\
\hline 7.985479 & 5.162600 & 5.222834 \\
\hline 6.079363 & 3.702500 & 4.480704 \\
\hline 5.488618 & 3.405187 & 3.604934 \\
\hline 7.055069 & 4.714447 & 4.235211 \\
\hline 4.283733 & 4.174012 & 10.082288 \\
\hline 3.393056 & 0.947392 & 7. 323521 \\
\hline 2.963400 & 0.825709 & 8.319395 \\
\hline 3.160079 & 4.464185 & 10.992821 \\
\hline 3.063392 & 0.003991 & 6.315187 \\
\hline 4.591776 & 1.182651 & 4.780448 \\
\hline 5.088437 & 1.242353 & 3.810529 \\
\hline 7.008382 & 5.228748 & 2.853909 \\
\hline 3.673353 & 0.126162 & 5.015285 \\
\hline 9.098269 & 6.144724 & 4.943768 \\
\hline 6.026932 & 5.538477 & 11.431688 \\
\hline 3.313801 & 5.603966 & 11.720777 \\
\hline 2.254712 & 5.891466 & 12.668131 \\
\hline 7.586309 & 6.450711 & 2.695689 \\
\hline 7.600558 & 6.968694 & 1.341492 \\
\hline 2.461326 & -1.858060 & 4.272905 \\
\hline 1.864061 & -1.980119 & 5.553132 \\
\hline 3.349648 & -0.829637 & 4.013196 \\
\hline 2.160966 & -1.069105 & 6.551411 \\
\hline 7.123532 & 5.541174 & 11.467476 \\
\hline 5.617945 & 5.170992 & 12.379913 \\
\hline 5.674121 & 6.573641 & 11.312713 \\
\hline 9.591525 & 5.966333 & 3.981306 \\
\hline 9.826100 & 6.073140 & 5.761896 \\
\hline 8.700046 & 7.169862 & 4.911618 \\
\hline 7.571738 & 2.056405 & 7.848793 \\
\hline 7.311641 & 1.311042 & 8.944856 \\
\hline 6.693204 & 1.785902 & 9.707657 \\
\hline 8.328539 & 1.511728 & 6.871459 \\
\hline 8.510142 & 2.145574 & 6.002512 \\
\hline 7.789537 & 0.012283 & 9.101994 \\
\hline 8.845015 & 0.220569 & 6.951502 \\
\hline 8.568027 & -0.549821 & 8.085329 \\
\hline 7.547346 & -0.547464 & 10.005771 \\
\hline 9.448775 & -0.172019 & 6.132853 \\
\hline 8.952782 & -1.566144 & 8.175539 \\
\hline 5.340678 & 5.813659 & 7.105718 \\
\hline 4.131414 & 5.816876 & 6.515951 \\
\hline 3.726411 & 4.839811 & 6.250193 \\
\hline 5.887870 & 6.993134 & 7.456081 \\
\hline 6.868499 & 6.941896 & 7.931355 \\
\hline 3.425347 & 6.989997 & 6.252072 \\
\hline 5.252175 & 8.213814 & 7.229867 \\
\hline 3.996912 & 8.212490 & 6.615228 \\
\hline 2.448281 & 6.940872 & 5.771897 \\
\hline 5.734942 & 9.143688 & 7.530371 \\
\hline 3.472958 & 9.149659 & 6.422526 \\
\hline 2.561781 & 6.816132 & 13.166421 \\
\hline 2.155880 & 5.075708 & 13.396258 \\
\hline 1.299925 & 6.035322 & 12.146595 \\
\hline 8.122649 & 7.927847 & 1.409089 \\
\hline 6.576393 & 7.113964 & 0.974734 \\
\hline 8.137046 & 6.284003 & 0.671831 \\
\hline 1.166980 & -2.797100 & 5.749242 \\
\hline 2.219247 & -2.581350 & 3.491898 \\
\hline 3.812923 & -0.735152 & 3.028261 \\
\hline 1.702118 & -1.161282 & 7.538513 \\
\hline
\end{tabular}

Table S11: low spin $\left[\mathrm{Fe}^{\mathrm{PA}} \mathbf{L}(\mathbf{p y})_{2}\right]$ (singlet; $\mathrm{COSMO}(\mathrm{MeCN})$ )

$\begin{array}{llll}\mathrm{Fe} & 6.061246 & 3.979677 & 7.312052 \\ \mathrm{O} & 7.596870 & 4.951663 & 6.649730 \\ \mathrm{O} & 6.448272 & 4.734827 & 9.049297 \\ \mathrm{~N} & 4.549133 & 3.021604 & 7.929856\end{array}$




\begin{tabular}{|c|c|c|c|}
\hline 0 & 7.112948 & 4.245155 & 1.953118 \\
\hline O & 2.545713 & 3.402223 & 11.457244 \\
\hline $\mathrm{N}$ & 5.651935 & 3.229744 & 5.621145 \\
\hline C & 4.092940 & 2.068000 & 7.006680 \\
\hline $\mathrm{C}$ & 5.673819 & 4.772290 & 10.069005 \\
\hline C & 3.948621 & 3.230787 & 9.087077 \\
\hline $\mathrm{H}$ & 3.016011 & 2.700042 & 9.301261 \\
\hline C & 4.713848 & 2.188466 & 5.699227 \\
\hline C & 7.894211 & 5.190525 & 5.426849 \\
\hline C & 6.156654 & 3.644207 & 4.473557 \\
\hline $\mathrm{H}$ & 5.774762 & 3.214297 & 3.542811 \\
\hline C & 7.168725 & 4.626132 & 4.323039 \\
\hline C & 4.400706 & 4.107370 & 10.105746 \\
\hline C & 3.179398 & 1.062950 & 7.255182 \\
\hline $\mathrm{H}$ & 2.728938 & 0.926805 & 8.237997 \\
\hline C & 3.453873 & 4.208377 & 11.234470 \\
\hline C & 2.816121 & 0.151347 & 6.227874 \\
\hline C & 4.388248 & 1.304796 & 4.689208 \\
\hline $\mathrm{H}$ & 4.859711 & 1.353444 & 3.708235 \\
\hline C & 7.441938 & 4.952997 & 2.909499 \\
\hline C & 3.431800 & 0.278549 & 4.912137 \\
\hline C & 9.113209 & 6.062782 & 5.253330 \\
\hline C & 6.249582 & 5.519357 & 11.246637 \\
\hline O & 3.629835 & 5.315081 & 12.004703 \\
\hline C & 2.745772 & 5.433906 & 13.147579 \\
\hline 0 & 8.057692 & 6.151898 & 2.730626 \\
\hline C & 8.388047 & 6.491662 & 1.360297 \\
\hline C & 2.216405 & -1.535052 & 4.172987 \\
\hline C & 1.609041 & -1.665203 & 5.482429 \\
\hline $\mathrm{C}$ & 0.661031 & -2.705644 & 5.704302 \\
\hline $\mathrm{H}$ & 0.214097 & -2.790182 & 6.696270 \\
\hline C & 0.327071 & -3.570478 & 4.684459 \\
\hline C & 0.920838 & -3.439780 & 3.395433 \\
\hline $\mathrm{C}$ & 1.844111 & -2.448070 & 3.144048 \\
\hline $\mathrm{H}$ & 2.308757 & -2.333664 & 2.163203 \\
\hline $\mathrm{H}$ & 0.637735 & -4.135186 & 2.603639 \\
\hline $\mathrm{H}$ & -0.400438 & -4.364845 & 4.859688 \\
\hline $\mathrm{N}$ & 3.124366 & -0.567397 & 3.909918 \\
\hline $\mathrm{N}$ & 1.922351 & -0.821096 & 6.492207 \\
\hline $\mathrm{H}$ & 7.325655 & 5.646528 & 11.074811 \\
\hline $\mathrm{H}$ & 6.076484 & 4.999928 & 12.197284 \\
\hline $\mathrm{H}$ & 5.783533 & 6.511600 & 11.332325 \\
\hline $\mathrm{H}$ & 9.775409 & 5.703394 & 4.456795 \\
\hline $\mathrm{H}$ & 9.649752 & 6.089390 & 6.209293 \\
\hline $\mathrm{H}$ & 8.814114 & 7.085836 & 4.984337 \\
\hline $\mathrm{N}$ & 7.216507 & 2.423763 & 7.726057 \\
\hline C & 7.114953 & 1.779899 & 8.914474 \\
\hline $\mathrm{H}$ & 6.389785 & 2.178412 & 9.620759 \\
\hline C & 8.105082 & 1.951940 & 6.818651 \\
\hline $\mathrm{H}$ & 8.160779 & 2.486232 & 5.871879 \\
\hline C & 7.883710 & 0.663972 & 9.228829 \\
\hline C & 8.905369 & 0.840552 & 7.064505 \\
\hline C & 8.795969 & 0.176324 & 8.289514 \\
\hline $\mathrm{H}$ & 7.756975 & 0.184728 & 10.199562 \\
\hline $\mathrm{H}$ & 9.599357 & 0.502911 & 6.295016 \\
\hline $\mathrm{H}$ & 9.406350 & -0.700649 & 8.506242 \\
\hline $\mathrm{N}$ & 4.952268 & 5.543720 & 6.922174 \\
\hline C & 3.734602 & 5.448572 & 6.331135 \\
\hline $\mathrm{H}$ & 3.392866 & 4.446404 & 6.081408 \\
\hline C & 5.407322 & 6.781392 & 7.247208 \\
\hline $\mathrm{H}$ & 6.386278 & 6.812342 & 7.719515 \\
\hline C & 2.949752 & 6.561101 & 6.049348 \\
\hline C & 4.674427 & 7.935711 & 6.993809 \\
\hline $\mathrm{C}$ & 3.420548 & 7.834162 & 6.383472 \\
\hline $\mathrm{H}$ & 1.979903 & 6.420779 & 5.572673 \\
\hline $\mathrm{H}$ & 5.090307 & 8.902754 & 7.276493 \\
\hline $\mathrm{H}$ & 2.826540 & 8.723936 & 6.173304 \\
\hline $\mathrm{H}$ & 3.054610 & 6.354897 & 13.652127 \\
\hline $\mathrm{H}$ & 2.861662 & 4.570502 & 13.815769 \\
\hline $\mathrm{H}$ & 1.701159 & 5.507838 & 12.818425 \\
\hline $\mathrm{H}$ & 8.895673 & 7.459030 & 1.421280 \\
\hline $\mathrm{H}$ & 7.476350 & 6.572776 & 0.754875 \\
\hline $\mathrm{H}$ & 9.052606 & 5.733662 & 0.925580 \\
\hline
\end{tabular}


Table S12: high spin [Fe $\left.{ }^{\mathrm{PA}} \mathrm{L}(\mathrm{py})_{2}\right]$ (quintet; $\mathrm{COSMO}(\mathrm{MeCN})$ )

\begin{tabular}{|c|c|c|c|}
\hline $\mathrm{Fe}$ & 6.421409 & 3.880914 & 7.462041 \\
\hline 0 & 7.926672 & 4.691762 & 6.413270 \\
\hline O & 6.510966 & 4.429006 & 9.388281 \\
\hline $\mathrm{N}$ & 4.621327 & 2.969211 & 7.980913 \\
\hline O & 6.479457 & 4.646456 & 1.880621 \\
\hline 0 & 2.124147 & 3.775698 & 11.110860 \\
\hline $\mathrm{N}$ & 5.744600 & 3.182647 & 5.618760 \\
\hline C & 4.207509 & 2.018830 & 7.049526 \\
\hline $\mathrm{C}$ & 5.583160 & 4.672631 & 10.242891 \\
\hline $\mathrm{C}$ & 3.873996 & 3.302795 & 9.014121 \\
\hline $\mathrm{H}$ & 2.846813 & 2.920701 & 9.068096 \\
\hline C & 4.828806 & 2.139072 & 5.737983 \\
\hline C & 7.971338 & 5.135385 & 5.208275 \\
\hline $\mathrm{C}$ & 6.037173 & 3.722254 & 4.452434 \\
\hline $\mathrm{H}$ & 5.449675 & 3.430604 & 3.572987 \\
\hline $\mathrm{C}$ & 7.031391 & 4.711562 & 4.216705 \\
\hline C & 4.254596 & 4.170395 & 10.074562 \\
\hline C & 3.331626 & 0.982239 & 7.308754 \\
\hline $\mathrm{H}$ & 2.894704 & 0.846450 & 8.298326 \\
\hline C & 3.142743 & 4.463741 & 11.003592 \\
\hline C & 3.003291 & 0.037971 & 6.298420 \\
\hline $\mathrm{C}$ & 4.539381 & 1.220559 & 4.747033 \\
\hline $\mathrm{H}$ & 5.027300 & 1.267333 & 3.773323 \\
\hline $\mathrm{C}$ & 7.008888 & 5.227063 & 2.831736 \\
\hline C & 3.620549 & 0.162493 & 4.982675 \\
\hline $\mathrm{C}$ & 9.112880 & 6.081664 & 4.929824 \\
\hline $\mathrm{C}$ & 6.029552 & 5.472757 & 11.441878 \\
\hline O & 3.310104 & 5.607117 & 11.718372 \\
\hline $\mathrm{C}$ & 2.267069 & 5.904917 & 12.681776 \\
\hline O & 7.590396 & 6.446592 & 2.688787 \\
\hline C & 7.631987 & 6.969889 & 1.336021 \\
\hline $\mathrm{C}$ & 2.481933 & -1.713612 & 4.276670 \\
\hline C & 1.872840 & -1.840056 & 5.584301 \\
\hline $\mathrm{C}$ & 0.966812 & -2.912681 & 5.823884 \\
\hline $\mathrm{H}$ & 0.517815 & -2.993899 & 6.815255 \\
\hline C & 0.674710 & -3.813637 & 4.821704 \\
\hline $\mathrm{C}$ & 1.270522 & -3.686973 & 3.534194 \\
\hline C & 2.154426 & -2.663192 & 3.266505 \\
\hline $\mathrm{H}$ & 2.620425 & -2.551287 & 2.285945 \\
\hline $\mathrm{H}$ & 1.021900 & -4.410702 & 2.756214 \\
\hline $\mathrm{H}$ & -0.020754 & -4.633091 & 5.011008 \\
\hline $\mathrm{N}$ & 3.352199 & -0.714538 & 3.997147 \\
\hline $\mathrm{N}$ & 2.146410 & -0.961864 & 6.578287 \\
\hline $\mathrm{H}$ & 7.125908 & 5.450601 & 11.476126 \\
\hline $\mathrm{H}$ & 5.613091 & 5.093453 & 12.382112 \\
\hline $\mathrm{H}$ & 5.699238 & 6.517596 & 11.345062 \\
\hline $\mathrm{H}$ & 9.598231 & 5.888641 & 3.966142 \\
\hline $\mathrm{H}$ & 9.839418 & 5.987092 & 5.746554 \\
\hline $\mathrm{H}$ & 8.745477 & 7.118318 & 4.899439 \\
\hline $\mathrm{N}$ & 7.498025 & 2.025431 & 7.808669 \\
\hline C & 7.226423 & 1.273891 & 8.897438 \\
\hline $\mathrm{H}$ & 6.613504 & 1.748500 & 9.664767 \\
\hline C & 8.250365 & 1.482242 & 6.827310 \\
\hline $\mathrm{H}$ & 8.443101 & 2.121986 & 5.965215 \\
\hline C & 7.688105 & -0.032023 & 9.042612 \\
\hline C & 8.750759 & 0.184208 & 6.896077 \\
\hline C & 8.461553 & -0.593005 & 8.021834 \\
\hline $\mathrm{H}$ & 7.437306 & -0.597442 & 9.940315 \\
\hline $\mathrm{H}$ & 9.351482 & -0.207736 & 6.075072 \\
\hline $\mathrm{H}$ & 8.833798 & -1.614704 & 8.103000 \\
\hline $\mathrm{N}$ & 5.341448 & 5.831678 & 7.111500 \\
\hline C & 4.130642 & 5.871340 & 6.525863 \\
\hline $\mathrm{H}$ & 3.702482 & 4.908406 & 6.246079 \\
\hline C & 5.916789 & 6.992975 & 7.478561 \\
\hline $\mathrm{H}$ & 6.897576 & 6.912843 & 7.949492 \\
\hline C & 3.450789 & 7.064211 & 6.283137 \\
\hline C & 5.308003 & 8.230716 & 7.273758 \\
\hline C & 4.051056 & 8.267400 & 6.663662 \\
\hline $\mathrm{H}$ & 2.471469 & 7.044645 & 5.805703 \\
\hline $\mathrm{H}$ & 5.812793 & 9.144443 & 7.587021 \\
\hline $\mathrm{H}$ & 3.547902 & 9.219096 & 6.487649 \\
\hline $\mathrm{H}$ & 2.584583 & 6.832793 & 13.166884 \\
\hline $\mathrm{H}$ & 2.180110 & 5.094562 & 13.417208 \\
\hline $\mathrm{H}$ & 1.304705 & 6.045882 & 12.174043 \\
\hline $\mathrm{H}$ & 8.155369 & 7.927061 & 1.417507 \\
\hline
\end{tabular}


6.615047

8.179159

7.118813

6.284949

0.951632

0.675591 